\title{
Temporal Dynamics of Antidepressant Ketamine Effects on Glutamine Cycling Follow Regional Fingerprints of AMPA and NMDA Receptor Densities
}

\author{
Meng Li ${ }^{1,2}$, Liliana Ramona Demenescu' ${ }^{1,2}$, Lejla Colic ${ }^{1,3}$, Coraline Danielle Metzger ${ }^{4,5,6,7}$, \\ Hans-Jochen Heinze $2,3,6,7$, Johann Steiner ${ }^{6,7}$, Oliver Speck ${ }^{3,4,6,8}$, Anna Fejtova ${ }^{3,6,9}$, Giacomo Salvadore ${ }^{10}$ and \\ Martin Walter ${ }^{*}, 1,3,6,7,11$ \\ 'Clinical Affective Neuroimaging Laboratory, Leibniz Institute for Neurobiology, Otto von Guericke University, Magdeburg, Germany; ${ }^{2}$ Department of \\ Neurology, Otto von Guericke University, Magdeburg, Germany; ${ }^{3}$ Leibniz Institute for Neurobiology, Magdeburg, Germany; ${ }^{4}$ German Centre for \\ Neurodegenerative Diseases (DZNE), Site Magdeburg, Germany; ${ }^{5}$ Institute of Cognitive Neurology and Dementia Research (IKND), Magdeburg, \\ Germany; ${ }^{6}$ Centre for Behavioural Brain Sciences (CBBS), Magdeburg, Germany; ${ }^{7}$ Department of Psychiatry and Psychotherapy, Otto von Guericke \\ University, Magdeburg, Germany; ${ }^{8}$ Department of Biomedical Magnetic Resonance, Otto von Guericke University, Magdeburg, Germany; ${ }^{9}$ Department \\ of Psychiatry and Psychotherapy, University Hospital, Friedrich-Alexander-University Erlangen-Nuremberg, Erlangen, Germany; ${ }^{10}$ Janssen Research and \\ Development, Titusville, NJ, USA; ' Department of Psychiatry and Psychotherapy, University of Tübingen, Tübingen, Germany
}

\begin{abstract}
The anterior cingulate cortex (ACC) has shown decreased glutamate levels in patients with major depressive disorder. Subanesthetic doses of ketamine were repeatedly shown to improve depressive symptoms within $24 \mathrm{~h}$ after infusion and this antidepressant effect was attributed to increased $\alpha$-amino-3-hydroxyl-5-methyl-4-isoxazole-propionate (AMPA) throughput. To elucidate ketamine's mechanism of action, we tested whether the clinical time course of the improvement is mirrored by the change of glutamine/glutamate ratio and if such effects show a regional and temporal specificity in two distinct subdivisions of ACC with different AMPA/N-methyl-D-aspartate receptor profiles. In a double-blind, placebo-controlled intravenous infusion study of ketamine, we measured glutamate and glutamine in the pregenual ACC (pgACC) and the anterior midcingulate cortex at I and $24 \mathrm{~h}$ post infusion with magnetic resonance spectroscopy at $7 \mathrm{~T}$. A significant interaction of time, region, and treatment was found for the glutamine/glutamate ratios (placebo, $n=14$; ketamine, $n=12$ ). Posthoc analyses revealed that the glutamine/glutamate ratio increased significantly in the ketamine group, compared with placebo, specifically in the pgACC after $24 \mathrm{~h}$. The glutamine/glutamate increase in the pgACC caused by ketamine at $24 \mathrm{~h}$ post infusion was reproduced in an enlarged sample (placebo, $n=24$; ketamine, $n=20$ ). Our results support a significant temporal and regional response in glutamine/ glutamate ratios to a single subanesthetic dose of ketamine, which mirrors the time course of the antidepressant response and reversal of the molecular deficits in patients and which may be associated with the histoarchitectonical receptor fingerprints of the ACC subregions. Neuropsychopharmacology (2017) 42, I20I-1209; doi:I0.1038/npp.2016.184; published online 19 October 2016
\end{abstract}

\section{INTRODUCTION}

The importance of the glutamatergic system in the pathophysiology and therapy of mood disorders (Krystal et al, 2002; Sanacora et al, 2008) has been recently supported by rapid antidepressant effects following a single intravenous subanesthetic dose of ketamine, a non-selective $N$-methyl-Daspartate (NMDA) receptor antagonist (Berman et al, 2000; Salvadore and Singh, 2013; Zarate et al, 2006). Initial

*Correspondence: Professor M Walter, Clinical Affective Neuroimaging Laboratory, Leibniz Institute for Neurobiology, Otto von Guericke University, Building 65, Leipziger Strasse 44, 39120 Magdeburg, Germany, Tel: +49 391 61 17 530, Fax: +49 391 61 17 531, E-mail: martin.walter@med.ovgu.de or Department of Psychiatry and Psychotherapy, University of Tübingen, Osianderstrasse 24, 72076 Tübingen, Germany, E-mail: martin.walter@uni-tuebingen.de

Received 7 January 2016; revised 26 August 2016; accepted 29 August 2016; accepted article preview online 8 September 2016 evidence suggests that ketamine acutely increases glutamate release, which leads to enhanced glutamatergic transmission (Maeng et al, 2008) and ultimately results in increased synaptic plasticity and synaptogenesis (Duman and $\mathrm{Li}$, 2012). However, ketamine's downstream effects have not been thoroughly investigated in humans. It is unknown whether changes in the glutamatergic activity following ketamine administration can be detected using available imaging methods and whether these changes show regional and/or specific temporal dynamics.

The antidepressant response to ketamine administration was associated with the pretreatment activity in the pregenual anterior cingulate cortex (pgACC) in treatment-resistant depressed patients (Salvadore et al, 2009; Salvadore and Zarate, 2010). This is in accordance with previous studies, which showed that increased pretreatment activity in the pgACC identifies responders to pharmacological antidepressant interventions (Chen et al, 2007a; Mayberg et al, 1997; Pizzagalli 
et al, 2001). These findings highlight the key role of the pgACC in antidepressant effects and the potential role of its activity as a biomarker of early treatment response in depression.

Studies in depression have reported abnormalities in glutamatergic metabolites in multiple brain regions using ${ }^{1} \mathrm{H}$ magnetic resonance spectroscopy (MRS) (Luykx et al, 2012; Yüksel and Öngür, 2010a). Recently, a study in major depressive disorder (MDD) patients revealed an increase in the glutamate + glutamine (glx) levels in the medial prefrontal cortex during ketamine infusion (Milak et al, 2015). In particular, in MDD patients, the pgACC is characterized by reduced glx (Auer et al, 2000; Yüksel and Öngür, 2010b), which is potentially reflective of a hypoglutamatergic state. Moreover, evidence from the direct measurements of glutamine concentrations further pointed toward an impaired neuron-glia interactions in the pgACC (Brennan et al, 2010; Walter et al, 2009). In addition, reduced levels of the astroglial glutamine synthetase have been found in the cingulate cortex of depression patients (Bernstein et al, 2015). Furthermore, an MRS study reported metabolic deficits in the pgACC, characterized in a subset of highly anhedonic MDD patients, predominantly showing reduced glutamine but not glutamate concentrations relative to the healthy volunteers or to the non-anhedonic patients (Walter et al, 2009). Given that such clinical phenomenology may predispose patients towards treatment resistance (Kautzky et al, 2015), a direct reversal of such a glutaminergic deficit was proposed as a putative mechanism of action of novel glutamatergic agents, especially those effective in treatmentresistant patients (Brennan et al, 2010; Walter et al, 2014).

Glutamine levels have been suggested as a marker for synaptically active glutamate and excitatory neurotransmission (Chowdhury et al, 2015; Hancu and Port, 2011). Changes in glutamine levels may be a particularly sensitive measure for detecting acute downstream effects following ketamine administration. Although it is possible to evaluate glutamate and glutamine separately, most early studies primarily focused on one of these two metabolites or pooled as ' $\mathrm{glx}$ '. In this study we explicitly focus on their respective ratio and thus follow a protocol, which was previously shown to be able to measure both substances at the same time (Dou et al, 2013).

ACC subdivisions can be delineated based on histoarchitectonic and receptor fingerprint markers (Palomero-Gallagher et al, 2009; Vogt et al, 2003). Although the pgACC has been found to exhibit above average $\alpha$-amino-3-hydroxyl-5-methyl4-isoxazole-propionate (AMPA) receptors and below average NMDA receptor densities (compared with whole cingulate cortex), the inverse has been reported for the anterior midcingulate cortex (aMCC) (Palomero-Gallagher et al, 2009). In addition, the aMCC and the pgACC have been proposed to have different roles in the regulation of mental and emotional activity interacting with other cortical and subcortical structures (Bush et al, 2000; Disner et al, 2011). A growing consensus indicates that depression is associated with dysfunction in these two ACC subregions (Chen et al, 2007b; Li et al, 2014; Pizzagalli, 2011). However, the ketamine effects in the pgACC and in the aMCC have not been directly compared in the scope of ketamine's reported antidepressant efficacy.

Importantly, most studies investigating neuronal and molecular effects of ketamine were performed during ketamine infusion. The transient psychometric effects are frequently found during the administration of ketamine and shortly afterwards (Luckenbaugh et al, 2014; Sos et al, 2013; Zarate et al, 2006). Therefore, measuring ketamine's effects only during or soon after its administration might be misleading, as it is hard to interpret whether findings reflect ketamine's acute dissociative effects or more specifically on antidepressant properties.

Here we assessed whether the reported time course of clinical improvement in patients could be mirrored by increased but delayed glutamine/glutamate ratios in healthy controls after ketamine infusion. We also tested for different pattern of regional and temporal dynamics of glutamine/ glutamate ratio.

\section{MATERIALS AND METHODS}

\section{Study Design}

A double-blind, randomized placebo-controlled study was conducted to assess the effects of a single intravenous infusion of ketamine on the brain metabolites in two ACC subregions (the pgACC and the aMCC) at two post infusion timepoints ( 1 and $24 \mathrm{~h}$ ). Given the observed ketamine's delayed effects on pgACC resting state activity in healthy individuals (Scheidegger et al, 2012, 2016), we focused on the neuronal mechanisms in carefully selected controls, without past or a family history of neuropsychiatric disorders in first-degree relatives.

This study was approved by the Institutional Review Board of the University of Magdeburg. All subjects gave written informed consent to participate after reviewing detailed written information about the study.

\section{Participants}

The study was conducted at the University Clinic of Magdeburg, enrolling a total of 58 healthy subjects, aged 19 to 50 years (mean: $26.48 \pm 5.66$ years, 34 males). The determination of the number of participants and the process of randomization are described in the Supplementary Materials and Methods. All subjects were in good physical state as determined by physical examination, medical history, blood laboratory tests, and electrocardiography. All subjects completed the mini-international neuropsychiatric interview, to ensure the absence of any ICD-10 psychiatric disorders. Subjects were free of comorbid substance abuse or dependence. In addition, all subjects underwent an eye examination to exclude ocular hypertension.

\section{Study Medication}

Study medication was supplied in identical $50 \mathrm{ml}$ syringes containing a clear solution of either $0.9 \%$ saline or $0.5 \mathrm{mg} / \mathrm{kg}$ body weight racemic ketamine (Ratiopharm GmbH, Ulm, Germany) with added saline to total $50 \mathrm{ml}$ of liquid volume. The infusion was administered continuously over $40 \mathrm{~min}$ via an infusion pump (Injectomat 2000, Fresenius Kabi GmbH, Langenhagen, Germany).

\section{Magnetic Resonance Image Acquisition}

In vivo magnetic resonance (MR) acquisitions were performed before the infusion (baseline), as well as 1 and $24 \mathrm{~h}$ after the start of infusion. 

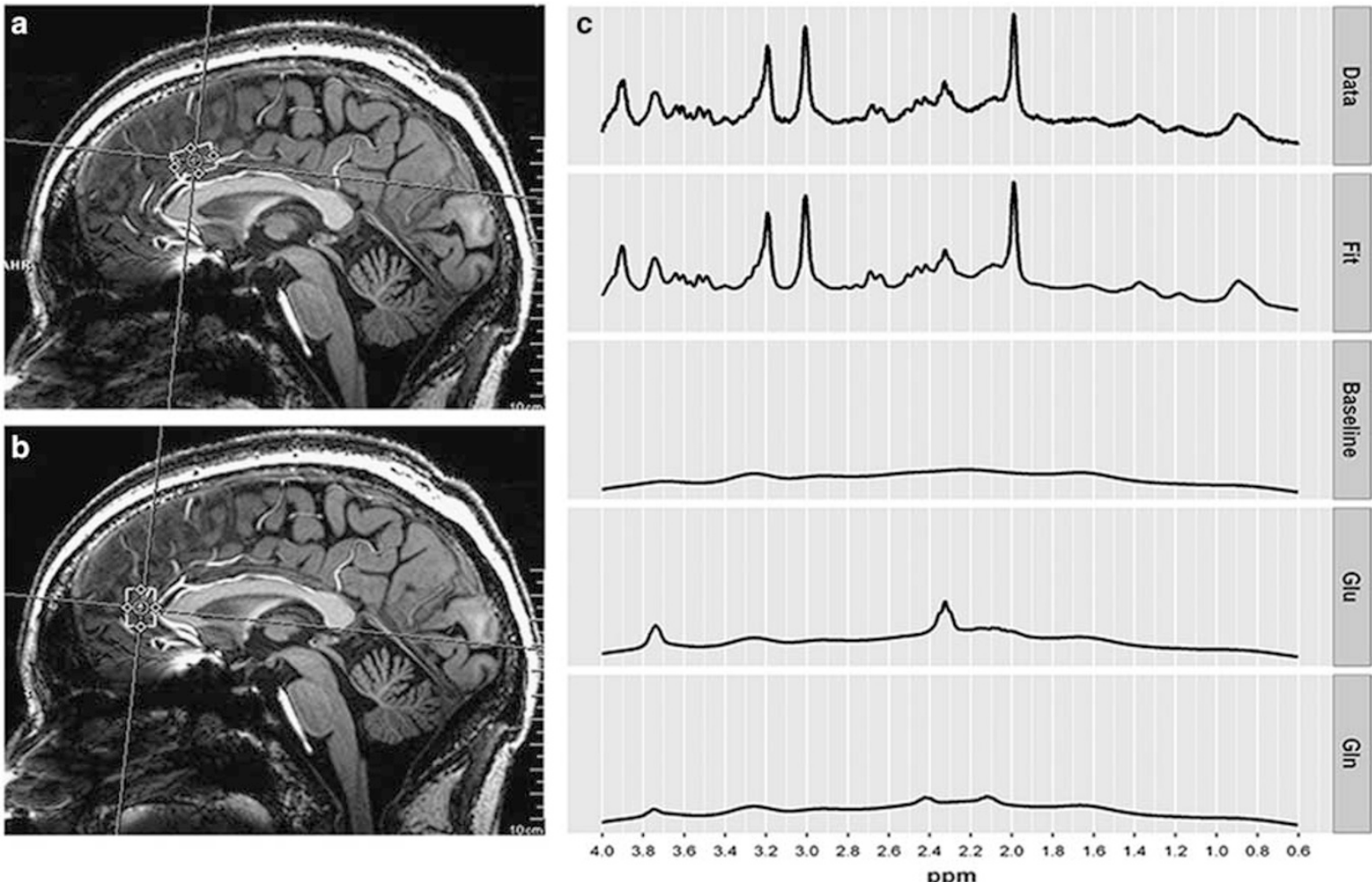

Figure I Magnetic resonance spectroscopy (MRS) voxel locations and exemplary spectrum showing metabolites and the corresponding model fit for our metabolites of interest. The anterior midcingulate cortex (aMCC, a) and pregenual anterior cingulate cortex (pgACC, b) voxel positioning in yellow and the exemplary spectrum (c) acquired from a study participant, showing the raw spectrum, the corresponding model fit, and baseline from LCModel analysis. A full color version of this figure is available at the Neuropsychopharmacology journal online.

MR images were acquired on a $7 \mathrm{~T}$ MR scanner (Siemens Healthcare, Erlangen, Germany) using a 32-channel head array coil. After an automated global shim, high-resolution T1weighted images were acquired with the following parameters: MPRAGE sequence, echo time $(\mathrm{TE})=2.73 \mathrm{~ms}$, repetition time $(\mathrm{TR})=2300 \mathrm{~ms}$, inversion time $(\mathrm{TI})=1050 \mathrm{~ms}$, flip angle $=5^{\circ}$, bandwidth $=150 \mathrm{~Hz} /$ pixel, acquisition matrix $=320 \times 320 \times$ 224 , and isometric voxel size $=0.8 \mathrm{~mm}^{3}$.

For the MRS acquisition, region-specific shimming was performed using an optimized vendor-provided, double-gradient echo shim technique. Following localized shimming, spectra were acquired from $25 \times 15 \times 10 \mathrm{~mm}^{3}$ voxel containing the aMCC (see Figure 1a) and from $20 \times$ $15 \times 10 \mathrm{~mm}^{3}$ voxel containing the pgACC (see Figure 1b). As shown in Figure 1a, the location of the aMCC voxel was defined as the center of the voxel projected to the posterior border of the genu of the corpus callosum and the location of the pgACC voxel (Figure 1b) was defined as bordering the lower edge of the genu of the corpus callosum and posteriorly touching the anterior border of the genu of the corpus callosum. Proton spectra were acquired using a stimulated-echo acquisition mode (STEAM) sequence with optimized variable rate selective excitation RF pulses (Elywa et al, 2012). The scan parameters included 128 averages, $\mathrm{TR}=3000 \mathrm{~ms}, \mathrm{TE}=20 \mathrm{~ms}, \mathrm{TM}=10 \mathrm{~ms}$, data size $=2048$, and bandwidth $=2800 \mathrm{~Hz}$. It took $6 \mathrm{~min}$ and $36 \mathrm{~s}$ for each region of interest. Water reference spectra were measured with one average for eddy current correction and served as an internal concentration reference for quantification. In addition, the MRS voxels during all three measurements were positioned in the same place for each subject individually, using a vendor-provided automatic voxel positioning technique (Dou et al, 2014).

LCModel (Stephen Provencher, Oakville, ON, Canada, Version 6.3.0) was applied to analyse all spectra (from 0.6 to 4.0 p.p.m.). The sequence-specific basis set for data analysis was measured and described in a previous study (Dou et al, 2013). The signals from macromolecules and lipids were simulated in LCModel using the method published by Seeger et al (2003). One example of a curve-fitting from a study participant is presented in Figure 1. The absolute concentrations of the target metabolites, ie, glutamine and glutamate, with respective Cramér-Rao Lower Bound (CRLB) and full width at half maximum (FWHM) values for spectral linewidth estimation were obtained. Metabolite concentrations were corrected for cerebrospinal fluid in investigated voxels using the cerebrospinal fluid fractions, obtained by segmenting the T1-weighted images with VBM8 (www. neuro.uni-jena.de/vbm/) in SPM8 (Wellcome Trust Centre for Neuroimaging, London, UK). Metabolite concentrations were expressed in institutional units. A recent study by Kreis (2016) suggested the absolute CRLB, instead of the relative CRLB, is appreciated to serve as an exclusion criterion for spectra analysis. To be specific, after screening the subjects with poor SNR $(\mathrm{SNR}<20)$ and spectral linewidth $(\mathrm{FWHM}>24 \mathrm{~Hz})$, the absolute CRLB of each concentration (glutamine or glutamate in both regions at three timepoints) was calculated in each subject. To every single concentration (glutamate or glutamine) in the same MRS voxel (aMCC or pgACC), the measurements at baseline were treated as 
control population for absolute CRLB computation. Next, the first and the third quartiles (Q1 and Q3), and the interquartile range (IQR) of absolute CRLB were calculated. Of them, the outliers were detected via boxplot and these subjects were excluded in the following analysis. Meanwhile, the individual absolute CRLB of each concentration at 1 and $24 \mathrm{~h}$ post infusion were tested against the threshold, which was defined as Q3 + 1.5 IQR of the corresponding concentration within control population. The subjects would be excluded if its value was larger than the derived threshold. With the resulting concentrations, subjects with abnormal values were detected and removed via boxplot. Our primary analysis only included subjects whose spectra survived in both investigated regions at all three timepoints. To overcome limited generalizability of post-hoc findings for this small sample, results were then validated in the region-specific validation data sets from all subjects. In the validation analysis, the spectra were screened from either pgACC or aMCC at all timepoints. In favor of readers interest, we also examined our spectra by replacing absolute CRLB with relative CRLB in the exclusion criteria and reported the results in Supplementary Materials.

\section{Statistical Analysis}

First, the concentration of glutamine and glutamate after partial volume correction for cerebrospinal fluid and the ratios of glutamine to glutamate were calculated for each subject.

Primary outcome measures were the derived glutamine to glutamate ratios at three timepoints in the aMCC and the pgACC. Repeated-measures analyses of variance (ANOVA) was used for the statistical analysis with glutamine/glutamate ratio as the dependent variable, region (aMCC or pgACC) and time (baseline, 1, or $24 \mathrm{~h}$ ) as the within-subject variables, and drug (ketamine or placebo) as the between-subject variable. The main effects for region, time, and drug, and their interactions are reported. Group comparison on the change of glutamine/glutamate at 1 and $24 \mathrm{~h}$ post infusion over baseline in each region was investigated via post-hoc two sample $t$-test. Likewise, repeated-measures ANOVA was also applied for the statistical analysis of glutamate and glutamine, respectively.

In the secondary analyses, the change of glutamine/ glutamate to its baseline after the infusion in pgACC, as well as the changes in aMCC, was investigated at both timepoints in each group. Meanwhile, the difference of the changes between pgACC and aMCC were also compared.

To confirm the effects revealed in primary analysis, we performed separate analyses of all the data available for the pgACC or the aMCC. The difference of the glutamine/ glutamate ratios between the ketamine and the control groups was tested at 1 and $24 \mathrm{~h}$. In the case of such (between subject) effects of drug, paired $t$-tests were used to investigate the (within subject) change of the glutamine/glutamate over baseline at $1 \mathrm{~h}$ and $24 \mathrm{~h}$ in the respective groups. In addition, the contribution of individual glutamine and glutamate levels to the ratio calculation (glutamine/glutamate) $24 \mathrm{~h}$ after ketamine infusion were investigated using a Pearsons product moment correlation.

All reported $p$-values are two-tailed. Results were considered significant at $p<0.05$. All statistical analyses were performed in $\mathrm{R}$ version 3.1.2 (www.r-project.org).

\section{RESULTS}

\section{Spectra Screened by Absolute CRLB}

A total of 58 subjects were randomized in the study. Twenty-nine of them failed to meet spectra quality criteria in at least one measurement according to the curve fitting, the $\mathrm{SNR}$, and/or the spectral linewidth. Two subjects were excluded due to abnormal absolute CRLB, compared with the derived value in the control population. One subject was further excluded, because the abnormal concentration in aMCC. After exclusion, 12 subjects $(28.0 \pm 8.1$ years, 5 female $)$ in the ketamine group and 14 subjects $(27.5 \pm 6.6$ years, 5 female $)$ in the placebo group were included in the primary analysis. Ketamine and placebo groups did not differ significantly in age $(t(21.3)=-0.198, p=0.844)$ or sex $\left(\chi^{2}(1, n=26)=0, p=0.755\right)$. Mean non-CSF tissue proportion, SNR, linewidth, CRLB, levels of glutamine and glutamate (after correction for the cerebrospinal fluid fraction), and the calculated glutamine/ glutamate in the aMCC and the pgACC from all three timepoints are listed in Table 1.

Primary analysis. A significant interaction of time, region, and treatment was found $(\mathrm{F}(2,96)=5.483, p=0.005)$ on the glutamine/glutamate ratio. Significant main effects was found for time $(\mathrm{F}(2,96)=6.307, p=0.002)$ and region $(\mathrm{F}(1,24)=48.072, \quad p<0.001)$, but not for treatment $(\mathrm{F}(1,24)=0.077, p=0.784)$. The results of the repeatedmeasurement ANOVA for glutamine and glutamate are reported in the Supplementary Materials.

Region and time specificity of ketamine effect on the glutamine/glutamate ratio. In the pgACC, the change in the glutamine/glutamate ratio at $24 \mathrm{~h}$ post ketamine infusion compared with baseline was significant $(t(11)=4.136$, $p=0.001$, see Figure 2), which is also significantly larger than the change observed at $24 \mathrm{~h}$ in the placebo group $(t(23.3)=2.618, p=0.015$, see Figure 2). However, the metabolite levels at $1 \mathrm{~h}$ after infusion in the same region in ketamine group was not significant different from the ones at baseline $(t(11)=1.482, p=0.166)$, as well as the difference of changes between ketamine and placebo at $1 \mathrm{~h}(t(22.1))=$ $-0.487, p=0.630)$, compared with their baseline level. In contrast, the changes between the ketamine and the placebo group in the aMCC were not significant at 1 or $24 \mathrm{~h}$ post infusion $(1 \mathrm{~h}: t(15.9)=0.456, p=0.654 ; 24 \mathrm{~h}: t(22.7)=$ $-0.523, p=0.605)$. The changes in the glutamine/glutamate ratio in aMCC from baseline in ketamine group $(1 \mathrm{~h}$ $t(11)=1.237, p=0.241 ; 24 \mathrm{~h}, t(11)=0.978, p=0.349)$ was not significant.

\section{Secondary analyses}

Regional dissociation. To investigate differential effects of local changes in the glutamine/glutamate ratios in the two subregions, we directly compared relative changes between the two ACC subdivisions. Only after $24 \mathrm{~h}$, the pgACC showed a larger change in the glutamine/glutamate ratio relative to the baseline when compared with the changes in the aMCC. This effect was restricted to the ketamine group only $(t(1)=3.691, p=0.003)$. After $1 \mathrm{~h}$, this effect had not developed yet in the ketamine group $(t(11)=0.198$, $p=0.846$ ) and no such effect was observed in the placebo 
Table I Mean Tissue Portion, SNR, Linewidth, and CRLB of Gln and Glu in aMCC and pgACC at Three Investigated Timepoints (Placebo: $n=14$; Ketamine, $n=12$ )

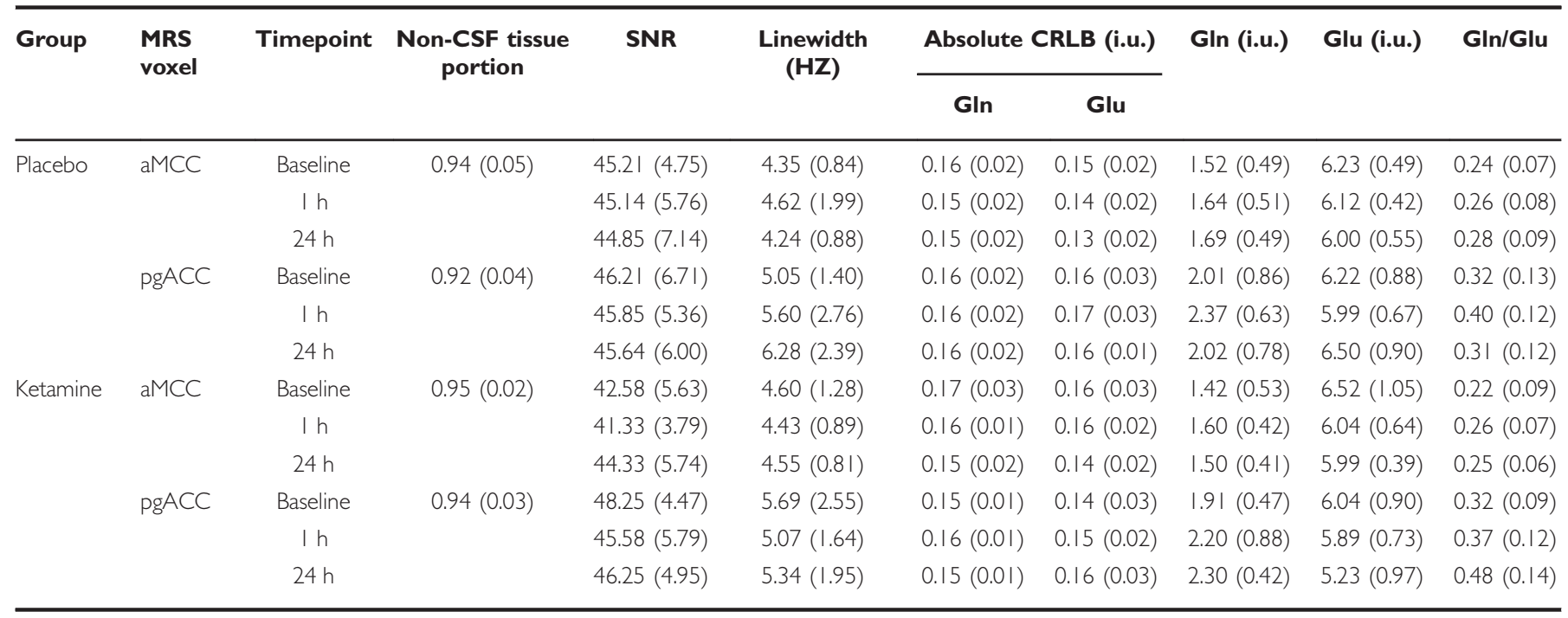

Abbreviations: aMCC, anterior middle cingulate cortex; CRLB, Cramér-Rao lower bound; Gln, glutamine; Glu, glutamate; i.u., institutional units; pgACC, pregenual anterior cingulate cortex.

All values were expressed using mean $\pm \mathrm{SD}$.

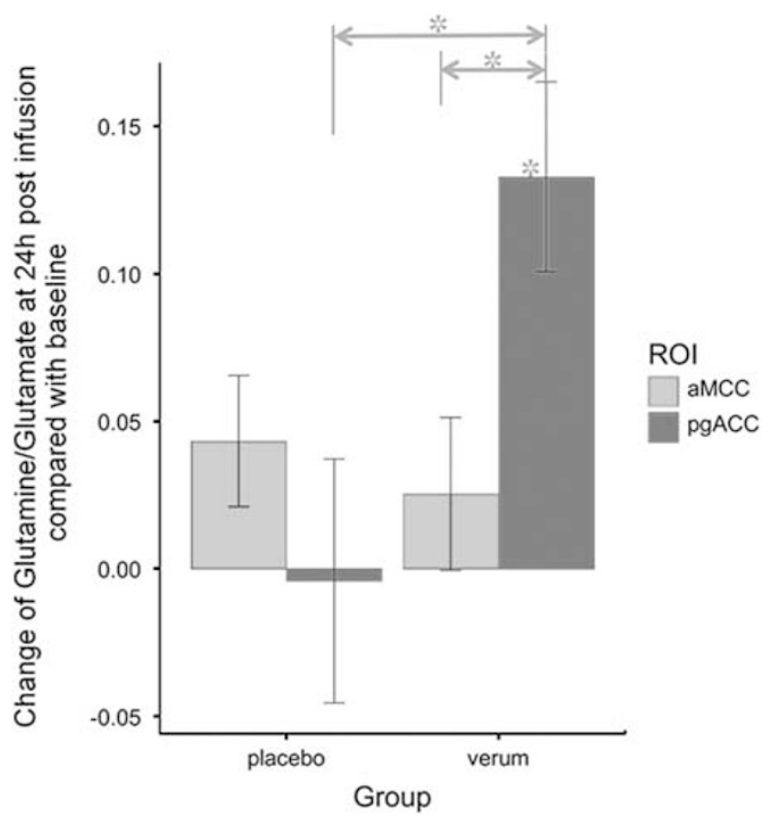

Figure 2 Results of regional comparison from the primary analysis. The change of glutamine/glutamate ratio at $24 \mathrm{~h}$ after intravenous ketamine infusion over baseline in the two anterior cingulate cortex subregions (aMCC, anterior middle cingulate cortex, in red; pgACC, pregenual anterior cingulate cortex, in dark green) in placebo $(n=\mid 4$, saline) and verum groups ( $n=12$, ketamine), respectively. Asterisks denote statistically significant changes in glutamine/glutamine relative to baseline levels. Error bars denote one SEM. A full color version of this figure is available at the Neuropsychopharmacology journal online.

group ( $1 \mathrm{~h}$ post infusion $>$ baseline: $t(13)=1.194, p=0.253$; $24 \mathrm{~h}$ post infusion $>$ baseline: $t(13)=-0.980, p=0.344)$.

Validation analysis. To confirm the effects revealed in the primary analysis, we performed separate analyses for data acquired in a larger sample. We had comparable datasets with 20 versus 24 subjects for the ketamine and the placebo group in the pgACC and 16 versus 15 subjects in the aMCC in the validation analysis.

The changes of the glutamine/glutamate ratio in the pgACC between ketamine and placebo groups was confirmed, that is significant difference $(t(39.9)=2.469, p=0.017$, see Figure 3 ) after $24 \mathrm{~h}$ but not in the pgACC after $1 \mathrm{~h}(t(41.7)=0.472$, $p=0.639)$. The difference between the placebo and ketamine after $24 \mathrm{~h}$ was driven by increased glutamine/glutamate ratio in the ketamine group $(t(19)=3.072, p<0.006$; see Figure 3 ) compared to the baseline and no change in the placebo subjects $(t(23)=-0.238, p<0.813)$.

In the ketamine subjects $24 \mathrm{~h}$ after infusion, the glutamine/ glutamate ratio in the pgACC showed a significant negative correlation with glutamate $(r=-0.751, p<0.001)$ and a significant positive correlation with glutamine levels $(r=0.904, p<0.001$; Figure 4).

In contrast, even with the larger sample size, comprising of all subjects with acceptable spectra in the aMCC, we did not find any effects of ketamine on the glutamine/glutamate ratio (group comparison of the change of the glutamine/glutamate against baseline: $24 \mathrm{~h}, t(28.2)=-0.457, p<0.651 ; 1 \mathrm{~h}$, $t(24.1)=0.356, p<0.724)$.

\section{DISCUSSION}

In the present study, a significant interaction of time, region, and treatment on the glutamine/glutamate ratio was found after ketamine infusion in healthy subjects. A follow-up analysis revealed significantly increased glutamine/glutamate ratio in the ketamine compared with the placebo group in the pgACC (not the aMCC) and only after $24 \mathrm{~h}$ (not after $1 \mathrm{~h})$. This translated into a change of the balance between regions: the glutamine/glutamate ratio in the pgACC relative to the levels in the aMCC was significantly increased 


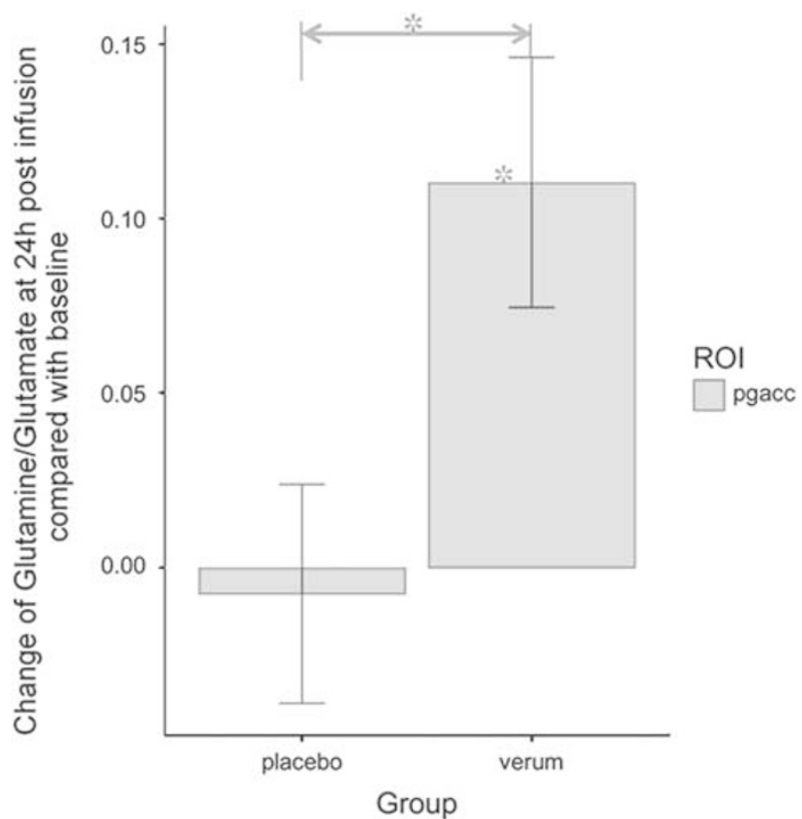

Figure 3 Validation of the effects in the pregenual anterior cingulate cortex (pgACC): significant increase in change of the glutamine/glutamate ratio after $24 \mathrm{~h}$ compared with the baseline in the ketamine group corresponds to a significant difference in the ketamine $(n=20)$ vs placebo $(n=24)$ in pgACC. Asterisks denote statistically significant change in the glutamine/glutamate relative to the baseline levels. Error bars denote SEM.

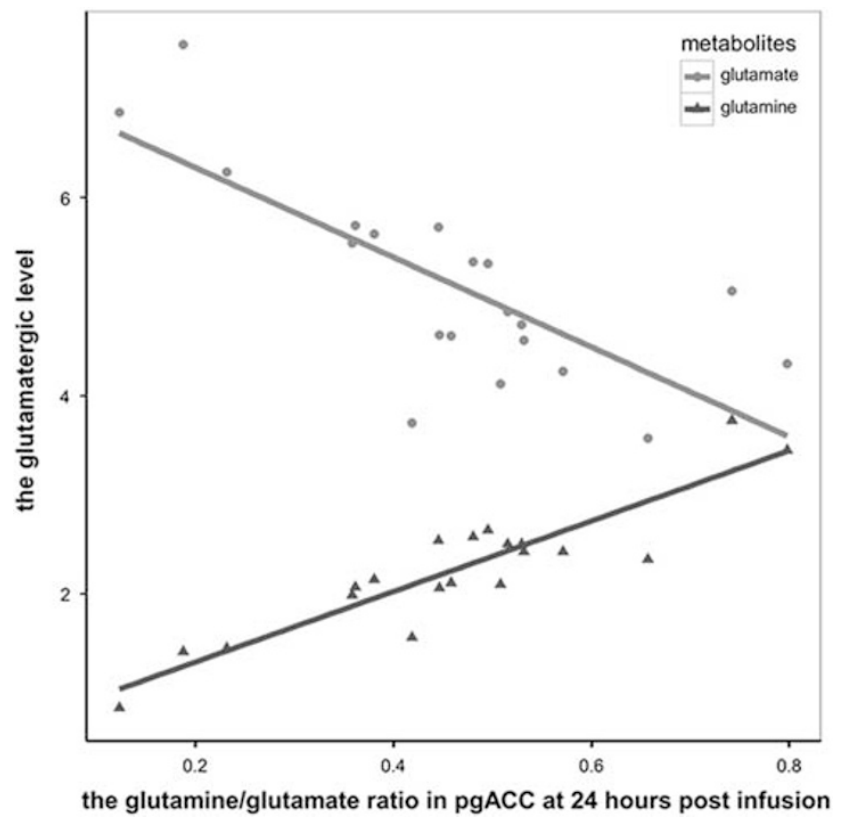

Figure 4 Correlation of the glutamine/glutamate ratio with the glutamate and the glutamine levels at $24 \mathrm{~h}$ post ketamine infusion $(n=20)$ in the validation analysis. The glutamine/glutamate ratios are predicted by the glutamate levels $(r=-0.75 \mathrm{I}, p<0.00 \mathrm{I})$ and the glutamine levels $(r=0.904$, $p<0.00$ I).

only $24 \mathrm{~h}$ after the ketamine infusion. This finding largely confirms our hypothesis of a regional specificity of ketamine effects on glutamatergic levels.

In an earlier study, Rowland et al (2005) reported evidence for acute glutamine (but not glutamate) changes following ketamine infusion. Rowland's finding was specific to the loading dose of ketamine rather than the maintenance dose afterwards. A recent study by Taylor et al (2012) investigating continuous infusion of subanesthetic ketamine dose did not find changes in the cortical glutamate or glx levels relative to baseline, during or at the end of the infusion in healthy volunteers. In contrast to these studies, our findings most closely match previous observations in a temporally similar study by Iltis et al (2009) who administered the NMDA antagonist phencyclidine to rats. They found a significant increase in glutamine/glutamate ratio compared with the baseline after phencyclidine infusion. Importantly, this ratio change, in the context of unchanged glx, was related to a strong increase of glutamine levels of $12 \%$ and a small decrease of glutamate levels (5\%), and increases of glutamine to glutamate ratios correlated with a reduction of glutamate and an increase of glutamine. These studies converge with the postulated reduced conversion of glutamine to glutamate underlying reduced glutamine/glutamate levels in depression (Yüksel and Öngür, 2010b). In an openlabel trial, Brennan et al (2010) investigated the effects of the glutamate modulating drug Riluzole on the glutamine/ glutamate ratio in ACC and parieto-occipital cortex in MDD patients. In their study, they found an increase in the glutamine/glutamate ratio 2 days after treatment. Moreover, this pattern of changes was more pronounced in the ACC than in the parieto-occipital cortex. In our study, using a placebo-controlled double-blind design, we demonstrate-if only for the healthy subjects-regional specificity within the ACC cortex. Our pgACC region not only substantially overlaps with the ACC region reported in Brennan et al (2010), but also addresses the margins of underlying histoarchitectonic maps (Palomero-Gallagher et al, 2009). Compellingly, these effects are observed both in direct comparisons between ketamine and the placebo as well as in comparison against the baseline.

In line with the importance of regional specificity, we demonstrated a significant difference of changes in glutamine/glutamate ratio in the pgACC relative to the aMCC after ketamine infusion. We hypothesized that this regional specificity is associated with AMPA receptor density and NMDA/AMPA receptor ratios in the respective regions (Walter et al, 2014). This hypothesis was based on observations showing that ketamine's antidepressant effects in MDD, which were expected to appear predominantly for the $24 \mathrm{~h}$, might rely on AMPA receptors (Maeng et al, 2008). Ketamine's antidepressant effect could be reversed by pretreatment with an AMPA antagonist (Koike et al, 2011) and blocked by the co-administration of an AMPA antagonist (Autry et al, 2011). A recent study suggested that the antidepressant actions by (R,S)-ketamine are independent of NMDA receptor inhibition but involve early and sustained activation of AMPA receptors (Zanos et al, 2016). The reduction of NMDA/AMPA receptor density ratios was further reported in the hippocampus of Wistar-Kyoto rats, a putative animal model of depression, after chronic treatment with an effective dose of ketamine (Tizabi et al, 2012). Interestingly, an opposite NMDA/AMPA receptor density ratio was reported in pgACC and aMCC (PalomeroGallagher et al, 2009). Furthermore, regional variations of glutamine and glutamate concentrations have been shown to follow receptor fingerprints of cingulate cortex in the human 
brain (Dou et al, 2013). Therefore, we were able to directly formulate a hypothesis on the regional specificity of receptor-related drug effects and their temporal profile incorporating mechanisms of homeostatic plasticity. The present data support our hypothesis that the pgACC had decreased glutamine/glutamate ratio in MDD (Walter et al, 2009; Yüksel and Öngür, 2010b), as well as high AMPA receptor densities, which resulted in an increase of glutamatergic ratios during antidepressant treatment (Brennan et al, 2010). In this view, the capacity of ketamine to reserve depressive symptoms on a similar timescale, ie, after 1-2 days, with efficacy dependent on AMPA-related mechanisms (Maeng et al, 2008).

Our findings describe novel neurochemical correlates of a subanesthetic ketamine infusion, which appear as late as $24 \mathrm{~h}$ after treatment. Scheidegger et al (2012) report the reduction of functional connectivity as a similar mechanism underlying ketamine's antidepressant efficacy, which may counteract the repeatedly observed hyperconnectivity within the default mode network in depressed patients. Although Scheidegger et al (2012) did not include earlier timepoints, it is intriguing that the parallel molecular changes seen in our study seem to build up until $24 \mathrm{~h}$ and only then do we see effects on crossregional balance of the metabolite ratios. The present results imply that changes in glutamine/glutamate are different from the acute brain effects during infusion of subanesthetic ketamine. The potential reversal of a pathologically reduced glutamine/glutamate levels as measured in terms of their ratios, as found in MDD, appears to be maximal after $24 \mathrm{~h}$ when the acute dissociative effects have vanished, but when the antidepressent effects are at their peak (Caddy et al, 2014; Fond et al, 2014; McGirr et al, 2015; aan het Rot et al, 2012).

Consequences for clinical translation need to be considered with caution, given that we only included healthy subjects in our study. Future studies are needed to broaden the time windows of observations especially the acute infusion stages. Moreover, after ketamine infusion in MDD patients, Milak et al (2015) have recently reported increased glx and GABA levels over baseline followed by normalization after $1 \mathrm{~h}$ in the medial prefrontal cortex. The first part of the acute response within the infusion period needs further confirmation at high field magnetic strength. The ideal next step would be to investigate glutamine/glutamate ratio changes both during and after infusion in patients with depression. Moreover, the fit for very low concentration metabolites such as glutamine via the metabolite basis set fitting program highly depends on what else is included in the basis set. Meanwhile, the ability to achieve reliable spectra quantification might be undermined by baseline distortion, with macromolecules as the main contributors, which is related to the TE values and hence can be a more critical issue at 7T (Jansen et al, 2006). Therefore, experimentally determined macromolecules should be applied and the robustness of estimated spectra and their potential overlaps still needs to be assessed in future studies.

In conclusion, we found that ketamine specifically increases glutamine/glutamate ratios in the pgACC, a region previously identified to display a deficit in these ratios in subjects with depression. This effect spatially reflects NMDA/AMPA receptor density profiles within the ACC subregions, which accordingly might be very relevant for ketamine's antidepressant properties. The molecular effects in the pgACC are also consistent with the change of interregional metabolite balance $24 \mathrm{~h}$ after ketamine administration, which ultimately may link to previously observed long-term functional network changes.

\section{FUNDING AND DISCLOSURE}

This work was supported by German Research Foundation (SFB 779/A06 for Drs Fejtova and Walter, and DFG Wa 2673/4-1 for Dr Walter), the Centre for Behavioural and Brain Sciences (CBBS NN05 for Drs Fejtova and Walter), and Leibniz Association (Park für Forschung und Innovation for Drs Fejtova and Walter). Dr Salvadore is employed at Janssen Research \& Development. Dr Walter has received research support from HEEL and Janssen Pharmaceutical Research. Dr Speck has received research support from Siemens Healthcare, which is not related to this study. Dr Li, Dr Demenescu, MS Colic, Dr Fejtova, Dr Metzger, Dr Heinze, and Dr Steiner declare no conflict of interest.

\section{ACKNOWLEDGMENTS}

We thank Renate Blobel and Dr Claus Tempelmann (Department of Neurology) for data acquisition; Dr Melanie Weigel (Department of Ophthalmology) and Dr Conrad Friedrich Genz (Department of Cardiology) for the clinical screening visits; Dr Jörn Kaufmann and Dr Weiqiang Dou for technical support on spectral data analysis; Dr Julia Noack and Linda Frolik Endrulat (Clinical Affective Neuroimaging Laboratory) for trial management; Dr Anton Lord and Viola Borchardt for biostatistical advice; and Dr Adam Safron and Dr Gina Joue for proofreading this manuscript.

\section{REFERENCES}

Auer DP, Pütz B, Kraft E, Lipinski B, Schill J, Holsboer F (2000). Reduced glutamate in the anterior cingulate cortex in depression: an in vivo proton magnetic resonance spectroscopy study. Biol Psychiatry 47: 305-313.

Autry AE, Adachi M, Nosyreva E, Na ES, Los MF, Cheng P et al (2011). NMDA receptor blockade at rest triggers rapid behavioural antidepressant responses. Nature 475: 91-95.

Berman RM, Cappiello A, Anand A, Oren DA, Heninger GR, Charney DS et al (2000). Antidepressant effects of ketamine in depressed patients. Biol Psychiatry 47: 351-354.

Bernstein H-G, Meyer-Lotz G, Dobrowolny H, Bannier J, Steiner J, Walter $\mathrm{M}$ et al (2015). Reduced density of glutamine synthetase immunoreactive astrocytes in different cortical areas in major depression but not in bipolar I disorder. Front Cell Neurosci 9: 273 .

Brennan BP, Hudson JI, Jensen JE, McCarthy J, Roberts JL, Prescot AP et al (2010). Rapid enhancement of glutamatergic neurotransmission in bipolar depression following treatment with riluzole. Neuropsychopharmacology 35: 834-846.

Bush G, Luu P, Posner MI (2000). Cognitive and emotional influences in anterior cingulate cortex. Trends Cogn Sci 4: 215-222.

Caddy C, Giaroli G, White TP, Shergill SS, Tracy DK (2014). Ketamine as the prototype glutamatergic antidepressant: pharmacodynamic actions, and a systematic review and meta-analysis of efficacy. Ther Adv Psychopharmacol 4: 75-99.

Chen C-H, Ridler K, Suckling J, Williams S, Fu CHY, Merlo-Pich E et al (2007a). Brain imaging correlates of depressive symptom 
severity and predictors of symptom improvement after antidepressant treatment. Biol Psychiatry 62: 407-414.

Chen C-H, Ridler K, Suckling J, Williams S, Fu CHY, Merlo-Pich E et al (2007b). Brain imaging correlates of depressive symptom severity and predictors of symptom improvement after antidepressant treatment. Biol Psychiatry 62: 407-414.

Chowdhury FA, O'Gorman RL, Nashef L, Elwes RD, Edden RA, Murdoch JB et al (2015). Investigation of glutamine and GABA levels in patients with idiopathic generalized epilepsy using MEGAPRESS. J Magn Reson Imaging 41: 694-699.

Disner SG, Beevers CG, Haigh EAP, Beck AT (2011). Neural mechanisms of the cognitive model of depression. Nat Rev Neurosci 12: 467-477.

Dou W, Palomero-Gallagher N, Tol M-J van, Kaufmann J, Zhong K, Bernstein H-G et al (2013). systematic regional variations of GABA, glutamine, and glutamate concentrations follow receptor fingerprints of human cingulate cortex. J Neurosci 33: $12698-12704$.

Dou W, Speck O, Benner T, Kaufmann J, Li M, Zhong K et al (2014). Automatic voxel positioning for MRS at 7 T. Magn Reson Mater Phys 28: 259-270.

Duman RS, Li N (2012). A neurotrophic hypothesis of depression: role of synaptogenesis in the actions of NMDA receptor antagonists. Phil Trans R Soc B 367: 2475-2484.

Elywa M, Mulla-Osman S, Godenschweger F, Speck O (2012). Proton magnetic resonance spectroscopy in deep human brain structures at 7 T. J Appl Spectrosc 79: 120-125.

Fond G, Loundou A, Rabu C, Macgregor A, Lançon C, Brittner M et al (2014). Ketamine administration in depressive disorders: a systematic review and meta-analysis. Psychopharmacology 231: 3663-3676.

Hancu I, Port J (2011). The case of the missing glutamine. NMR Biomed 24: 529-535.

Iltis I, Koski DM, Eberly LE, Nelson CD, Deelchand DK, Valette J et al (2009). Neurochemical changes in the rat prefrontal cortex following acute phencyclidine treatment: an in vivo localized ${ }^{1} \mathrm{H}$ MRS study. NMR Biomed 22: 737-744.

Jansen JF, Backes WH, Nicolay K, Kooi ME (2006). 1H MR spectroscopy of the brain: absolute quantification of metabolites 1 . Radiology 240: 318-332.

Kautzky A, Baldinger P, Souery D, Montgomery S, Mendlewicz J, Zohar J et al (2015). The combined effect of genetic polymorphisms and clinical parameters on treatment outcome in treatment-resistant depression. Eur Neuropsychopharmacol 25: 441-453.

Koike H, Iijima M, Chaki S (2011). Involvement of AMPA receptor in both the rapid and sustained antidepressant-like effects of ketamine in animal models of depression. Behav Brain Res 224: 107-111.

Kreis R (2016). The trouble with quality filtering based on relative Cramér-Rao lower bounds: the trouble with quality filtering based on relative CRLB. Magn Reson Med 75: 15-18.

Krystal JH, Sanacora G, Blumberg H, Anand A, Charney DS, Marek $\mathrm{G}$ et al (2002). Glutamate and GABA systems as targets for novel antidepressant and mood-stabilizing treatments. Mol Psychiatry 7 Suppl 1: S71-S80.

Li M, Metzger CD, Li W, Safron A, Tol M-J, van, Lord A et al (2014). Dissociation of glutamate and cortical thickness is restricted to regions subserving trait but not state markers in major depressive disorder. J Affect Disord 169: 91-100.

Luckenbaugh DA, Niciu MJ, Ionescu DF, Nolan NM, Richards EM, Brutsche NE et al (2014). Do the dissociative side effects of ketamine mediate its antidepressant effects? J Affect Disord 159: $56-61$.

Luykx JJ, Laban KG, Heuvel MP, van den, Boks MPM, Mandl RCW, Kahn RS et al (2012). Region and state specific glutamate downregulation in major depressive disorder: a meta-analysis of 1H-MRS findings. Neurosci Biobehav Rev 36: 198-205.
Maeng S, Zarate CA, Du J, Schloesser RJ, McCammon J, Chen $G$ et al (2008). Cellular mechanisms underlying the antidepressant effects of ketamine: role of $\alpha$-amino-3-hydroxy-5methylisoxazole-4-propionic acid receptors. Biol Psychiatry 63: 349-352.

Mayberg HS, Brannan SK, Mahurin RK, Jerabek PA, Brickman JS, Tekell JL et al (1997). Cingulate function in depression: a potential predictor of treatment response. Neuroreport 8: 1057-1061.

McGirr A, Berlim MT, Bond DJ, Fleck MP, Yatham LN, Lam RW (2015). A systematic review and meta-analysis of randomized, double-blind, placebo-controlled trials of ketamine in the rapid treatment of major depressive episodes. Psychol Med 45: 693-704.

Milak MS, Proper CJ, Mulhern ST, Parter AL, Kegeles LS, Ogden RT et al (2015). A pilot in vivo proton magnetic resonance spectroscopy study of amino acid neurotransmitter response to ketamine treatment of major depressive disorder. Mol Psychiatry 21: $320-327$.

Palomero-Gallagher N, Vogt BA, Schleicher A, Mayberg HS, Zilles K (2009). Receptor architecture of human cingulate cortex: evaluation of the four-region neurobiological model. Hum Brain Mapping 30: 2336-2355.

Pizzagalli D, Pascual-Marqui RD, Nitschke JB, Oakes TR, Larson CL, Abercrombie HC et al (2001). Anterior cingulate activity as a predictor of degree of treatment response in major depression: evidence from brain electrical tomography analysis. Am J Psychiatry 158: 405-415.

Pizzagalli DA (2011). Frontocingulate dysfunction in depression: toward biomarkers of treatment response. Neuropsychopharmacology 36: 183-206.

Rot M aan het, Zarate CA Jr., Charney DS, Mathew SJ (2012). Ketamine for depression: where do we go from here? Biol Psychiatry 72: 537-547.

Rowland LM, Bustillo JR, Mullins PG, Jung RE, Lenroot R, Landgraf $E$ et al (2005). Effects of ketamine on anterior cingulate glutamate metabolism in healthy humans: a 4 -T proton MRS study. Am J Psychiatry 162: 394-396.

Salvadore G, Cornwell BR, Colon-Rosario V, Coppola R, Grillon C, Zarate CA et al (2009). Increased anterior cingulate cortical activity in response to fearful faces: a neurophysiological biomarker that predicts rapid antidepressant response to ketamine. Biol Psychiatry 65: 289-295.

Salvadore G, Singh JB (2013). Ketamine as a fast acting antidepressant: current knowledge and open questions. CNS Neurosci Ther 19: 428-436.

Salvadore G, Zarate CA (2010). Magnetic resonance spectroscopy studies of the glutamatergic system in mood disorders: a pathway to diagnosis, novel therapeutics, and personalized medicine? Biol Psychiatry 68: 780-782.

Sanacora G, Zarate CA, Krystal JH, Manji HK (2008). Targeting the glutamatergic system to develop novel, improved therapeutics for mood disorders. Nat Rev Drug Discov 7: 426-437.

Scheidegger M, Henning A, Walter M, Boeker H, Weigand A, Seifritz E et al (2016). Effects of ketamine on cognition-emotion interaction in the brain. NeuroImage 124(Part A): 8-15.

Scheidegger M, Walter M, Lehmann M, Metzger C, Grimm S, Boeker $\mathrm{H}$ et al (2012). Ketamine decreases resting state functional network connectivity in healthy subjects: implications for antidepressant drug action. PLOS ONE 7: e44799.

Seeger U, Klose U, Mader I, Grodd W, Nägele T (2003). Parameterized evaluation of macromolecules and lipids in proton MR spectroscopy of brain diseases. Magn Reson Med 49: 19-28.

Sos P, Klirova M, Novak T, Kohutova B, Horacek J, Palenicek T (2013). Relationship of ketamine's antidepressant and psychotomimetic effects in unipolar depression. Neuroendocrinol Lett 34: 101-107. 
Taylor MJ, Tiangga ER, Mhuircheartaigh RN, Cowen PJ (2012). Lack of effect of ketamine on cortical glutamate and glutamine in healthy volunteers: a proton magnetic resonance spectroscopy study. J Psychopharmacol 26: 733-737.

Tizabi Y, Bhatti BH, Manaye KF, Das JR, Akinfiresoye L (2012). Antidepressant-like effects of low ketamine dose is associated with increased hippocampal AMPA/NMDA receptor density ratio in female Wistar-Kyoto rats. Neuroscience 213: 72-80.

Vogt BA, Berger GR, Derbyshire SWG (2003). Structural and functional dichotomy of human midcingulate cortex. Eur $J$ Neurosci 18: 3134-3144.

Walter M, Henning A, Grimm S, Schulte RF, Beck J, Dydak U et al (2009). The relationship between aberrant neuronal activation in the pregenual anterior cingulate, altered glutamatergic metabolism, and anhedonia in major depression. Arch Gen Psychiatry 66: 478-486.
Walter M, Li S, Demenescu LR (2014). Multistage drug effects of ketamine in the treatment of major depression. Eur Arch Psychiatry Clin Neurosci 264: 55-65.

Yüksel C, Öngür D (2010a). Magnetic resonance spectroscopy studies of glutamate-related abnormalities in mood disorders. Biol Psychiatry 68: 785-794.

Yüksel C, Öngür D (2010b). Magnetic resonance spectroscopy studies of glutamate-related abnormalities in mood disorders. Biol Psychiatry 68: 785-794.

Zanos P, Moaddel R, Morris PJ, Georgiou P, Fischell J, Elmer GI et al (2016). NMDAR inhibition-independent antidepressant actions of ketamine metabolites. Nature 533: 481-486.

Zarate CA, Singh JB, Carlson PJ, Brutsche NE, Ameli R, Luckenbaugh DA et al (2006). A randomized trial of an $\mathrm{N}$-methyl-D-aspartate antagonist in treatment-resistant major depression. Arch Gen Psychiatry 63: 856-864.

Supplementary Information accompanies the paper on the Neuropsychopharmacology website (http://www.nature.com/npp) 